\title{
Histological typing of lung and pleural tumours: third edition
}

The previous World Health Organisation (WHO) histological classifications of lung tumours (1967 and 1981) have been difficult to apply consistently and reproducibly. This has been particularly problematical for small biopsies. It has resulted in considerable difficulties in interpreting studies of the frequency of the various histological types in different countries and situations, and it is also important because histological type affects the type of treatment administered. These problems have been partly the result of the inadequate criteria provided for the accurate classification of tumours and partly because of the great diversity of patterns encountered in lung tumours. An example of this is the great variability in the published percentages for bronchioloalveolar carcinoma in different studies, which are mainly the result of using variable histological criteria for the diagnosis. The authors of this new WHO classification $^{1}$ of lung tumours are to be congratulated on tackling many of these issues, taking into account recent advances in biological knowledge of some of these tumours, and providing clear and firm criteria for classifying many of the tumours. Hopefully, this will lead to greater consistency in histological typing and make published studies easier to compare and interpret. The figures are of high quality and provide good examples of the various pulmonary lesions. Considerably more variants have been introduced since the previous classification, although there is only one addition to the major categories: lymphoproliferative diseases. Nevertheless, although greatly improved, we feel that there are still some weaknesses, inconsistencies, and imbalances within the classification and that there were some missed opportunities. For example, there is a long and detailed account of neuroendocrine lesions, with many variants, but very limited subdivisions of mesothelial and lymphoproliferative disorders. The old term "sclerosing hemangioma" coined by Liebow and Hubbell ${ }^{2}$ was retained and these tumours were put into the group of miscellaneous lesions, whereas the weight of evidence indicates that they are of epithelial origin and would have been placed more appropriately into the group of adenomas. ${ }^{3}$ Sclerosing hemangioma could reasonably be regarded as a variant of alveolar adenoma. The authors quite rightly stress that the classification relies predominantly on light microscopical appearances, but regrettably provide little guidance as to when histochemical and immunohistochemical stains should be applied; indeed, there is some discouragement for the use of mucin stains. We feel that the use of mucin stains should be routine because they are easy to perform and several solid adenocarcinomas, poorly differentiated adenocarcinomas, and adenosquamous carcinomas would be misclassified without their use. It would also have been useful to have provided guidance for the appropriate sampling of tumours because lung tumours are so frequently heterogeneous. It is important to recognise that lung cancers can be very heterogeneous. The $10 \%$ rule, although arbitrary, is very helpful for standardising classification. For example, adenosquamous carcinoma should only be diagnosed when each component comprises $10 \%$ or more of the tumour. Otherwise, most lung carcinomas could be diagnosed as mixed. This has implications for small biopsies, where heterogeneity may be missed because of the small size of the sample. Several studies have shown poor reproducibility of lung tumour classification on small biopsies, and one should not attempt too sophisticated a classification on these types of specimen. ${ }^{4}$ A simple distinction on biopsy between non-small cell and small cell carcinoma is often sufficient for treatment purposes.

The section on preinvasive lesions, a very difficult and confusing area, is very helpful and welcome. Squamous dysplasia/carcinoma in situ, atypical adenomatous hyperplasia, and a very rare entity, diffuse idiopathic neuroendocrine cell hyperplasia (DIPNECH), are included in this category. There is a clear table describing the architectural and cytonuclear features of the various grades of squamous dysplasia and carcinoma in situ. However, for the illustrations corresponding to the grades of dysplasia, the reader is referred to those of the cervix, whereas it would have been useful to have included illustrations in this publication. Studies on reproducibility and biological relevance are needed in this area. Although not specifically mentioned, it is implied that squamous metaplasia and dysplasia are precursor phases of squamous cell carcinoma. The earlier phases of metaplasia are thought to be reversible in most instances, whereas in severe dysplasia and carcinoma in situ there are frequently irreversible genetic mutations similar to those found in the adjacent squamous cell carcinoma. We are fully in agreement with the authors that atypical adenomatous hyperplasia (AAH) should not be subdivided into several grades, which some investigators have done, because of lack of reproducibility. The association between AAH and adenocarcinoma is mentioned but the biological relevance of a diagnosis of $\mathrm{AAH}$ is still far from clear and needs longitudinal follow up. We regret the introduction of the new criteria for bronchioloalveolar carcinoma: lepidic growth without invasion. To maintain consistency in biological and pathological terminology this should not have been included in the adenocarcinoma group but should have been placed under preinvasive lesions, although we recognise that this disease can be fatal owing to pulmonary functional impairment. Under the new WHO definition, this tumour will be very rarely diagnosed and there will be considerable uncertainty where atypical adenomatous hyperplasia ends and bronchioloalveolar carcinoma begins-diagnosis is dependent largely upon size and it is implied that $5 \mathrm{~mm}$ should be the "cut off" point. Although it is recognised that distinctly more spacing between cells may be present in adenoma, overlapping images can occur even at several sites within one lesion. DIPNECH is a very rare disorder, which may be associated with airway fibrosis and obstruction; multiple tumourlets, typical and atypical carcinoids are frequently present. In contrast no association with small cell carcinoma has been described. Interestingly, Auerbach et al described squamous metaplasia and dysplasia occurring in association with small cell carcinoma. ${ }^{5}$ In this respect, the bronchial epithelium seems morphologically to behave similarly in the development of squamous and small cell carcinoma, except for the unexplained "sudden" change to small cell carcinoma. The rarity of DIPNECH, and the relatively high incidence of small cell carcinoma, makes it unlikely that DIPNECH is a precursor of many small cell carcinomas. Because neuroendocrine bodies do not form in the normal adult lung, ${ }^{6}$ it is possible that the smoking related, potentially reversible, neuroendocrine cell hyperplasia in the form of neuroendocrine bodies could be a precursor lesion for small cell carcinoma.

The section on neuroendocrine tumours is detailed and comprehensive and somewhat out of balance with the other sections. Nevertheless, the criteria given for separating carcinoid tumours into typical and atypical are very 
welcome; as the authors point out, since the original description by Arrigoni et al, ${ }^{7}$ the diagnosis has sometimes been somewhat arbitrary and many alternative names have been given to these tumours. Most weight is given to mitotic activity and necrosis-typical carcinoids lack necrosis and show less than two mitoses $/ 2 \mathrm{~mm}^{2}$, atypical carcinoids show from two to nine mitoses $/ 2 \mathrm{~mm}^{2}$ (or the presence of punctate necrosis), and small cell carcinoma and large cell neuroendocrine carcinoma show 10 or more mitoses $/ 2 \mathrm{~mm}^{2}$ and often large areas of necrosis. This requires the pathologist to calibrate his/her microscope so that it is known how many high power fields correspond to this standard area. This should result in a reduction of interobserver variation and should be much easier to apply than relying on pleomorphism. A methodological weakness, irrespective of the calibration improvement to $\mathrm{mm}^{2}$, is the use of a one number threshold twice (for example, two or 10) as the decision point, because population statistics have not been taken into account. A grey area would have been preferred.

The authors provide cogent arguments for categorising well differentiated fetal adenocarcinoma as a variant of adenocarcinoma. Formerly it was considered as a pure epithelial form of pulmonary blastoma, a biphasic tumour, because the typical endometrioid epithelial pattern is seen in both tumours. The reasons provided are the better prognosis in well differentiated fetal adenocarcinoma and the lack of p53 mutations seen in pulmonary blastoma.

Mesothelial tumours, which appear to be increasing in incidence in several countries, are dealt with rather cursorily and the classification is rather limited. A surprising omission was the well differentiated papillary mesothelioma which, although very rare in the pleura, should be clearly separated from diffuse malignant mesothelioma because it has a much better prognosis and an absent or very weak association with asbestos exposure. Preinvasive lesions, which have been termed by various authors as atypical mesothelial hyperplasia or mesothelioma in situ, were not included either, in contrast to the fairly lengthy descriptions of preinvasive epithelial lesions.

The classification of lymphoproliferative lesions, a very complex subject, was also fairly limited. Neither post transplantation lymphoproliferative lesions nor the primary pleural effusion body cavity lymphomas were included.

We hope that the authors of the new WHO classification will not feel aggrieved by our criticisms and will accept them in the spirit in which they were given. We think that the third edition is a considerable improvement on the previous two editions of the WHO classification and should enable more consistent and reproducible reporting of lung and pulmonary tumours, which in turn should lead to better treatment and understanding of the biology of these diverse, complex, and fascinating tumours.

A R GIBBS

Department of Histopathology, Llandough Hospital, Cardiff Vale NHS Trust, Cardiff CF64 2XX, UK

F B J M THUNNISSEN

Department of Pathology, Canisius Wilhelmina Hospital, PO Box 9015, NL6500 GS Nijmegen, The Netherlands

1 Travis WD, Colby TV, Corrin B, et al, eds. Histological typing of lung and pleural tumours, 3rd ed. Berlin: Springer, 1999.

2 Liebow AA, Hubbell DS. Sclerosing hemangioma (histiocytoma, xan-

3 thoma) of the lung. Cancer 1956;9:53-75. Semeraro D, Gibbs AR. Pulmonary adenoma: a variant
haemangioma of the lung? $\mathcal{C}$ Clin Pathol $1989 ; 42: 1222-3$.

4 haemangioma of the lung? F Clin Pathol 1989;42:1222-3. St J Thomas J, Lamb D, Ashcroft T, et al. How reliable is the diagno
lung cancer using small biopsy specimens? Thorax 1993;48:1135-9.

5 Auerbach O, Hammond EC, Garfinkel L. Changes in bronchial epithelium in relation to cigarette smoking, $1955-1960$ vs. 1970-1977. N Engl f Med 1979;300:381-5.

6 Boers JE, den Brok JLM, Koudstaal J, et al. Number and proliferation of neuroendocrine cells in normal human airway epithelium. Am $\mathcal{f}$ Respir Crit Care Med 1996;154:758-63.

7 Arrigoni MG, Woolner LB, Bernatz PE. Atypical carcinoid tumors of the lung. I Thorac Cardiovasc Surg 1972;64:413-21. 